\title{
DEVELOPMENT OF KM-5 HALL EFFECT THRUSTER AND ITS FLIGHT TESTING ONBOARD GEO SPACECRAFT "EXPRESS-A4"
}

\author{
V. N. Akimov, S. G. Baidakov, V. N. Galayko, \\ O. A. Gorshkov, Y. M. Ermoshkin, V. A. Muravlev, \\ A. V. Nikipelov, A. A. Sinitsin, V. F. Petrusevitch, \\ V. N. Shutov, A. I. Vasin, and D. V. Volkov
}

\begin{abstract}
The paper presents the outcomes of development of Keldysh Research Center (KeRC) KM-5 Hall effect thruster and experience obtained from the in-orbit exploiting of the thruster onboard Reshetnev's Scientific and Production Association of Applied Mechanics (NPO PM) "Express-A4" GEO communication spacecraft (SC). KM-5 is a multimode thruster with operational power range from 1 to $2.5 \mathrm{~kW}$ in contrast to other thrusters applied in space missions. It has high thrust characteristics. It makes possible solution of orbit raising tasks to geostationary orbit (GEO) for medium and heavy weight SC in addition to tasks of station keeping for geostationary SC already being solved by Hall-effect thrusters. Furthermore, a thruster with such performance can be an efficient choice as a main engine for small interplanetary probes.
\end{abstract}

\section{INTRODUCTION}

Based on the predictions of COMSAT [1] for the forthcoming decade (20062015), $90 \%$ of communication SC launched to GEO (17-19 SC per year) will have the launching mass from 2.2 to 5.4 t and more (SC mass on GEO from 1.5 to $4.0 \mathrm{t}$ ). The approximate mass distribution of such spacecraft is given in Table 1. Active lifetime for commercial geostationary SC will be $\sim 10-15$ years.

A number of satellite platforms (SP) that are under operation or under development allow creation of geostationary communication SC with the mass on GEO of 2.5-4 t. Examples include Eurostar-2000+ (SC Astra 2B, Hot-bird-2, -7), Eurostar-3000 (Intelsat X-01, Anik F3, Inmarsat-4), Eurostar-4000, as well as SP Spacebus-3000 (AMC-9, Galaxy 17, Astra 1K) and Spacebus-4000 (AMC-12, $-22,-23$, Koreasat-5, APStar 6) for advanced SC, which are produced by Astrium and Alcatel. Nowadays, low-power bipropellant liquid rocket engines as well as electrical propulsion (EP), including Hall-effect thrusters and ion thrusters 
Table 1 Mass distribution of commercial GEO SC to be launched in 20062015 (forecast)

\begin{tabular}{cc}
\hline $\begin{array}{c}\text { SC mass } \\
\text { on GEO, t }\end{array}$ & $\begin{array}{c}\text { Fraction of total } \\
\text { GEO SC number, \% }\end{array}$ \\
\hline $1.3-2.5$ & 30 \\
$2.5-3.5$ & 40 \\
$>3.5$ & 20 \\
\hline
\end{tabular}

are applied as a part of SC stationkeeping propulsion system of geostationary SC. Application of EP with the specific impulse of $1500-1700$ s reduces the amount of propellant needed to execute power-consuming (especially, inclination control) maneuvers of GEO station keeping by a factor of approximately 56 relative to low-thrust liquid propulsion [2] with the specific impulse of $\sim 300 \mathrm{~s}$.

Hall-effect EP, mainly, SPT-100 with the power of $1.35 \mathrm{~kW}$ and the specific impulse of $1500 \mathrm{~s}$, developed by EDB "Fakel" are applied on Russian geostationary space platforms, and by foreign SC-producing companies: Alcatel and Astrium in Europe, as well as Lockheed Martin Astrospace in USA. Boeing Satellite Space company applies ion thrusters XIPS-13 with the power of $0.65 \mathrm{~kW}$ and XIPS-25 with the power of $2.3-4.5 \mathrm{~kW}$ and the specific impulse of $3200-3800 \mathrm{~s}$, respectively.

One of the trends in modern European SC development on the basis of the platforms mentioned above is the increase of SC mass with a corresponding increase of on-board electric system power.

This means that more electric power can be assigned to orbit correction system, so EP of higher power (with higher thrust value) can be applied for SC orbit correction. This, in turn, would allow decreasing the required time for SC fire operation, defined by total impulse.

With respect to the given features of SC development, a Hall-effect thruster KM-5 was created in KeRC [3]. The thruster is capable of effective operation in power range from 1 to $2.5 \mathrm{~kW}$. At the same time, thrust and specific impulse vary in the ranges of $52-140 \mathrm{mN}$ and $1560-2100 \mathrm{~s}$, respectively. At the nominal regime with power of $2 \mathrm{~kW}$, the thrust is $115 \mathrm{mN}$ with the specific impulse of 1700 s. In contrast to SPT-70 and SPT-100 HETs that are already applied in space, the KM-5 thruster developed by KeRC provides increased power, thrust, and specific impulse.

KM-5 application onboard the station keeping system of a GEO SC improves its thrust-to-mass ratio and decreases the required firing time if compared to SPT-100 application for the same task. In particular, if the KM-5 thruster operates at power of $2.5 \mathrm{~kW}$ with the thrust of $140 \mathrm{mN}$, its operation time required for a 4.5-ton GEO SC does not exceed $5000 \mathrm{~h}$, whereas the operation time required for SPT-100 is about $8000 \mathrm{~h}$. Such a decrease in the demanded lifetime may decrease the costs of thruster development and increase its reliability.

Higher power provides the KM-5 thruster with advanced capabilities in orbit raising of medium and heavy satellites to GEO. In this case, the payload mass of such SC can be significantly increased. The use of Hall thrusters within 
a 10-kilowatt EP system (EPS), particularly, 8-10 KM-5 units (including spare thrusters) having specific impulse of 1500-1700 s, even in a case of short operation time of 30-50 days, increases payload mass by $20 \%-30 \%$ in comparison with a direct injection to GEO by a chemical propulsion boost vehicle or an apogee liquid propulsion system. If the launch is performed from Baikonur launch site by a "Proton-M" launch vehicle with a chemical propulsion "DM-3" boost vehicle, the absolute value of payload mass increases by $100-200 \mathrm{~kg}$. If the SC power-tomass ratio on the GEO is $5 \mathrm{~kW} / \mathrm{t}$, EPS power is $\sim 20-25 \mathrm{~kW}$, and the injection time is increased up to 180 days, the payload mass can be increased by a factor of 1.5-2 as compared with chemical propulsion.

The Hall thrusters can also be used for aerodynamic drag compensation of low-orbit SC used for Earth remote sensing in order to prolong their active operation time (up to 5-10 years). If the EPS is equipped with Hall thrusters providing $\sim 100-120 \mathrm{mN}$ thrust and having lifetime of $6000 \mathrm{~h}$, it can keep a 300kilometer high orbit of a $\mathrm{SC}$ with mass equal to $1 \mathrm{t}$ and power-to-mass ratio of $\sim 2.5 \mathrm{~kW} / \mathrm{t}$. This allows one to increase the resolution of the SC optical system. In this case, the EPS spends an amount of xenon equal to $10 \%-15 \%$ of total SC mass while operating for 5 years with a specific impulse of $1700 \mathrm{~s}$.

Missions to planets of the solar system are among the reachable tasks for small SC with main EPS. For example, KM-5 thruster with power of $1.5-2 \mathrm{~kW}$ allows transferring an SC with $165 \mathrm{~kg}$ initial mass from low Earth orbit (LEO) to Mars orbit with a height of pericenter equal to $600 \mathrm{~km}$ and orbital period of two days. The SC mass on the Mars orbit would be $80-90 \mathrm{~kg}$, including dry EPS mass of about $35-40 \mathrm{~kg}$. The flight time will be less than 2 years.

Considering the prospect of development and possible commercial interest in such equipment by Russian and foreign customers, KeRC and NPO PM decided to perform flight qualification of KM-5 multimode thruster onboard "ExpressA4" SC in April 2001.

Keldysh Research Center performed manufacturing, testing, and delivery of the thruster and gas distribution unit (GDU). It also provided the models and documentation required to confirm the suitability of the equipment for installation onto the SC.

Reshetnev's Scientific and Production Association of Applied Mechanics performed integration of the thruster, supplying power, executive instructions, and propellant gas. The company was also responsible for combined checkups and coupling control. It also controls the thruster in orbit and collects telemetric data.

Since the power supply for KM-5 thruster onboard "Express-A4" satellite can be provided only by the standard power source, with output voltage of $300 \mathrm{~V}$ and discharge current of $4.5 \mathrm{~A}$, it was decided to operate in this mode, rather than in nominal mode (350 $\mathrm{V}$ and $5.7 \mathrm{~A}$ ). A special matching and control unit (MCU) was developed and manufactured by SPC "Polus" to provide power supply and control of the thruster. 
In this way, an additional propulsion unit (APU) applicable for "Express-A4" SC was developed by NPO PM based on KM-5 thruster and GDU.

\section{PROPULSION UNIT DESCRIPTION}

The propulsion unit developed for a flight test onboard "Express-A4" consists of the KM-5 thruster and a GDU. A photograph of the KM-5 thruster and the GDU is presented in Fig. 1.

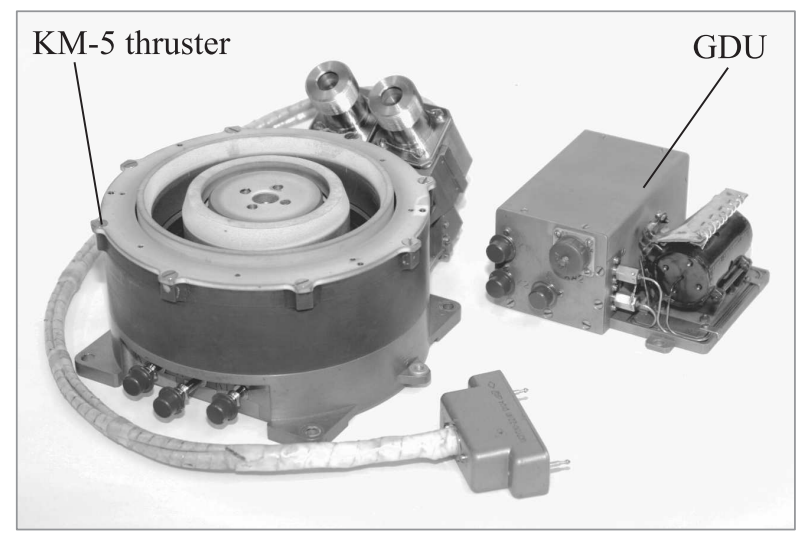

Figure 1 Hall thruster KM-5 and GDU

\subsection{Flight Unit Basic Specifications}

The basic specifications of the orbit-correction propulsion-unit flight model are presented in Table 2.

\subsection{Thruster}

Discharge chamber and walls of the acceleration channel are completely made of boron nitride based ceramics (hot-pressed mixture of boron nitride and silicon dioxide). The outer wall diameter of a cylindrical part of the acceleration channel is $116 \mathrm{~mm}$ and the channel width is $15.5 \mathrm{~mm}$.

Two hollow cathodes with $\mathrm{W}-\mathrm{Ba}$ emitters are used as neutralizers. One of them is a spare. Cathode startup is performed with preliminary emitter heating 
Table 2 Basic specifications of the propulsion-unit flight model

\begin{tabular}{lc}
\hline \multicolumn{1}{c}{ Parameter } & Value \\
\hline Discharge voltage, V & $300 \pm 15$ \\
Discharge current, A & $4.5 \pm 0.1$ \\
Thrust, mN & $80 \pm 4$ \\
Specific impulse, s & $\geq 1,550$ \\
Lifetime required for the experiment onboard "Express-A4," h & 4000 \\
Number of on/off cycles & 4000 \\
Thruster mass (cable included), kg & $\leq 4.9$ \\
GDU mass with filter-getter unit, kg & $\leq 0.8$ \\
\hline
\end{tabular}

Table 3 KM-5 thruster performance

\begin{tabular}{ccccc}
\hline Power, W & Voltage, V & Thrust, mN & Specific impulse, s & Efficiency, \% \\
\hline \multirow{2}{*}{1,350} & 300 & 82 & 1,570 & 47 \\
& 350 & 71 & 1,780 & 46 \\
& 450 & 65 & 1,830 & 43 \\
\hline \multirow{2}{*}{2,000} & 350 & 111 & 1,880 & 51 \\
& 450 & 102 & 1,970 & 49 \\
\hline \multirow{2}{*}{2,500} & 350 & 137 & 1,970 & 53 \\
& 450 & 128 & 2,090 & 52 \\
\hline
\end{tabular}

by an ohmic heater. The heater is also used for emitter outgassing before in-orbit thruster startup.

The thruster was primarily designed to have a multimode operation capability, to provide total impulse of $2.2 \times 10^{6} \mathrm{~N} \cdot \mathrm{s}$ and to operate at nominal power of $2000 \mathrm{~W}$. Additionally, it was designed to be capable of performing prolonged operation in de-rated power mode $(1350 \mathrm{~W})$ and to be capable of carrying out short runs at enhanced power of $2500 \mathrm{~W}$. The output parameters achieved in the course of optimization are as shown in Table 3.

\subsection{Gas Distribution Unit}

Gas distribution unit is responsible for xenon feed of the thruster and controls the magnitude of discharge current. Xenon runs from the propellant feed subsystem of the main propulsion system through the GDU to the KM-5. While the thruster is operating, xenon pressure at the GDU inlet is maintained at a level of 245 $\pm 20 \mathrm{kPa}$. The functional pneumatic diagram is presented in Fig. 2 .

The GDU has a main and spare xenon feed lines for both cathodes and the anode in order to increase its reliability. The required cathode flow rate is 


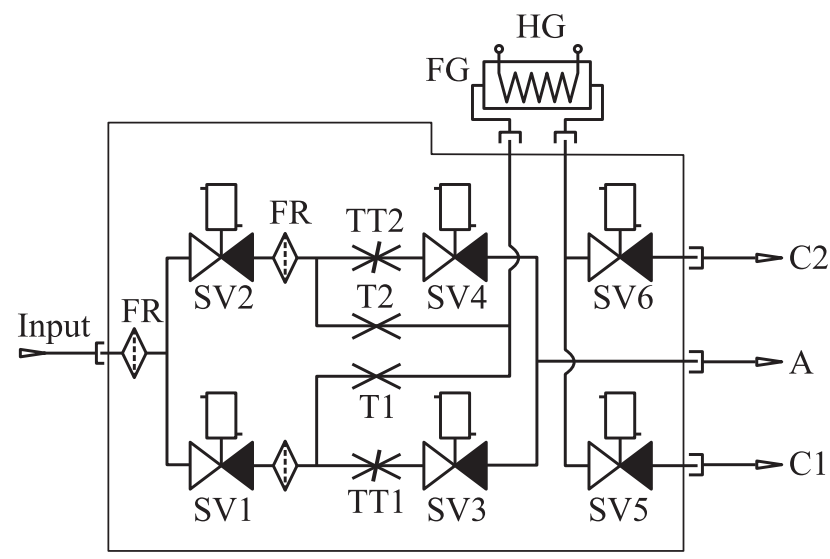

Figure 2 Gas distribution unit functional schematics: FR - filter; SV - electromagnetic valve; TT - thermal throttle; $\mathrm{T}$ - throttle; FG - filter-getter; $\mathrm{C}$ - cathode pipeline; A - anode pipeline; and HG - heater of the filter-getter

obtained by installation of a suitable throttle. Varying the magnitude of the current running through a thermal throttle according to a command from the matching and control unit maintains a given value of anode flow rate.

The cathode feed line is equipped with a filter-getter (FG) to protect the cathode emitters from poisoning by contaminants contained in xenon and structural material of the feed-line elements. Xenon passes through porous chemically pure titanium held in an ampoule of the FG at temperature of about $750{ }^{\circ} \mathrm{C}$. At that temperature, the contaminants in the propellant (mainly, oxygen and water) react with titanium and are absorbed by it as a result.

The power consumed by the FG heater during stationary operation is no more than $17 \mathrm{~W}$. The total power consumption by the GDU is no more than $23 \mathrm{~W}$.

The parts and structural units used as elements of the GDU and KM-5 thruster were subjected to a large number of tests in the course of T-160 and ROS-99 propulsion units development, including 500- and 2500-hour life tests, respectively.

\section{PROPULSION UNIT QUALIFICATION ACTIVITIES}

As the "Express-A4" onboard electric power of KM-5 can only be supplied by the standard onboard power source with output voltage of $300 \mathrm{~V}$ and discharge current of $4.5 \mathrm{~A}$, it was decided to carry out operation of the KM-5 in this mode, while using the discharge current to power magnetic coils. 
Taking into account features of onboard operation, the already manufactured engineering models of KM-5 and GDU were modified and separately put to mechanical tests with qualification load levels and functioning tests. Coupled firing tests of the KM-5 and GDU engineering models (EMs) were also performed. Plume measurements and temperature measurements of thruster parts were also carried out at that time. The plume measurements showed that $95 \%$ of ion flow passes within the limits of 58 degree divergence angle. The EM coupled test also allowed the electric filter parameters and magnitude of the thermal throttle current to be determined more exactly. According to the test results, EMs were accepted to coupled firing tests with the spacecraft equipment held at NPO PM.

In addition to functioning tests, qualification models (QM) of KM-5 and GDU were tested for resistance to mechanical and thermal effects. They were then subjected to a 500-hour life test.

\subsection{Mechanical and Environmental Tests}

The mechanical tests of KM-5 QM and GDU QM were performed separately. Load levels and duration are presented in Table 4. The linear acceleration was replaced by an equivalent sinusoidal effect. To determine the equipment condition before and after applying full-scale vibration loads, amplitude-frequency characteristics were studied. Control procedures were performed after the mechanical tests in order to check conformance to the requirements of design documents.

Table 4 Levels and durations of mechanical loads

\begin{tabular}{llc}
\hline \multicolumn{1}{c}{ Load type } & \multicolumn{1}{c}{ Parameters } & Values \\
\hline \multirow{3}{*}{ Sinusoidal vibration } & Frequency range, $\mathrm{Hz}$ & $5-100$ \\
& Acceleration, $g$ & $1-20$ \\
& Duration per axis, min & 10 \\
\hline \multirow{3}{*}{ Random vibration } & Spectral density, $g^{2} / \mathrm{Hz}$ & $0.002-0.133$ \\
& Duration per axis, min & 6 \\
& Variance $\sigma, g$ & 12.9 \\
\hline \multirow{2}{*}{ Linear acceleration } & Acceleration, $g$ & \pm 10 \\
& Duration per axis, min & 10 \\
\hline \multirow{3}{*}{ Shock } & Acceleration, $g$ & 45.3 \\
& Pulse duration, ms & 43 \\
& Number of shocks per axis & 6 \\
\hline \multirow{2}{*}{ Vibration endurance } & Frequency range, Hz & $5-200$ \\
& Acceleration, $g$ & $1-12$ \\
& Duration per axis, min & 30 \\
\hline
\end{tabular}


KM-5 QM examination procedure included its startup and operation at nominal power mode using main and spare cathodes, thrust, anode and cathode flow rates, and temperature measurements. In the course of thruster firing tests, vacuum facility backpressure varied in the range of $2 \cdot 10^{-4}-1 \cdot 10^{-2} \mathrm{~Pa}$.

Gas distribution unit examination procedure included a valve operation check, determination of flow rate value while operating with main and spare pipelines, determining of flow rate in cathode and anode gas circuits, measurement of thermal throttles standby current, pipeline pressurization check, and verification of conformance to design documents.

Thermal environment tests of KM-5 QM and GDU QM were designed to prove experimentally their resistance to cyclically changing heat loads in a vacuum environment.

KM-5 QM was subjected to 10 test thermal cycles in a qualification range of temperatures from the minimum $\left(-70{ }^{\circ} \mathrm{C}\right)$ to the maximum $\left(+160{ }^{\circ} \mathrm{C}\right)$ level. The range corresponds to "firing" operation onboard an SC. During the tests, the startup was performed at minimal $\left(-70^{\circ} \mathrm{C}\right)$ as well as at maximal $\left(+160^{\circ} \mathrm{C}\right)$ temperature. Each thermal cycle included cooling of the thruster down to $-70{ }^{\circ} \mathrm{C}$, exposure to this temperature for $2 \mathrm{~h}$ or longer, until thermal equilibrium was achieved, startup ("cold startup") and subsequent functioning for $180 \mathrm{~min}$ at $I=4.6 \mathrm{~A}$ and $U=315 \mathrm{~V}$, until the equilibrium temperature $\left(\sim 160{ }^{\circ} \mathrm{C}\right)$ was attained. After reaching this temperature, the thruster was switched off and after a 5-minute pause, started up again in "hot startup" and then operated for $90 \mathrm{~min}$. After that, the cycle was repeated.

While performing GDU QM thermal qualification activities, 10 cycles of negative $\left(-30^{\circ} \mathrm{C}\right)$ and positive $\left(+70{ }^{\circ} \mathrm{C}\right)$ temperature excursions were made to check the effect on the base part of GDU QM. The cycle duration was $\sim 150-170 \mathrm{~min}$ with GDU QM elements functioning. The tests included FG operation, xenon flow rate feed to the pipelines, and measurement of cathode throttle panel and GDU QM wall temperatures. Examinations of equipment condition after the tests showed that the performance of GDU QM elements was nominal. Leakages and overflows in valve couples were equal to the initially measured values.

Coupled firing tests were performed to verify equipment functionality after shock tests imitating transport loads as well as to check functionality in a case of discharge voltage, valve voltage, thermal throttle current, and xenon input pressure deviation from nominal values (within the specification limits). The thrust was in the range of $78.4-83.2 \mathrm{mN}$, and the specific impulse in a range of 1560-1600 s when the discharge voltage varied from 285 to $315 \mathrm{~V}$ and the GDU input pressure of xenon varied from 225 to $265 \mathrm{kPa}$. Anode current overload duration was less than $10 \mathrm{~ms}$ and its amplitude value was $4.8 \mathrm{~A}$.

Plume parameters, namely, ion energy distribution and divergence angle $\left(58^{\circ}-59^{\circ}\right)$, were measured at the end of the test. Thruster firing time totalled $47 \mathrm{~h}$ and 23 min during the course of qualification tests; the number of on/off cycles was 35 . 


\subsection{Life Test}

The 500-hour life test was divided into cycles. Each cycle lasted for $180 \mathrm{~min}$ (firing time $160 \mathrm{~min}$ ). Cathode heating time $(4 \mathrm{~min}$ ) was included into the duration of pause. During the test, the discharge voltage and current were permanently maintained at $300 \pm 6 \mathrm{~V}$ and $4.5 \pm 0.1 \mathrm{~A}$, respectively. A total of $188 \mathrm{on} / \mathrm{off}$ cycles were performed during the test, with 5 of them using the spare cathode.

Approximately every $100 \mathrm{~h}$ of operation, plume measurements were made and functioning tested using the spare cathode. Total firing time of the QM in the course of the life and qualification tests amounted to $554 \mathrm{~h}$ with $225 \mathrm{on} / \mathrm{off}$ cycles, from which 11 firings were performed after exposure in the atmosphere. During the tests, KM-5 QM switched on and operated together with the GDU QM without any failures.

Thrust measurement was performed at the end of each cycle, at the moment of thruster turnoff. Registration and measurement of other operating parameters of the thruster and the GDU were performed shortly before the turnoff. Calibration of the thrust measurement device (TMD) was performed after each thrust measurement since such a procedure eliminates errors caused by TMD zero drift.

In the course of the life test, the thruster parameters were stable (thrust $80 \pm 4 \mathrm{mN}$, specific impulse $\sim 1550 \mathrm{~s}$, power $\sim 1350 \mathrm{~W}$ ) and matched the specification. The histories of discharge voltage, discharge current, and thrust variation are presented in Fig. 3. Angular divergence did not change during the tests period and was equal to $56^{\circ}$.

Wall erosion measurements were made before the life test, then after 120 , 250,364 , and $507 \mathrm{~h}$ of operation. The profile of eroding parts of the insulator was measured at different distances from the channel exit, in 8 cross-sections, uniformly distributed through the azimuth. The profiles for 250, 364, and $507 \mathrm{~h}$ are presented in Fig. 4. Straight lines correspond to the initial profile.
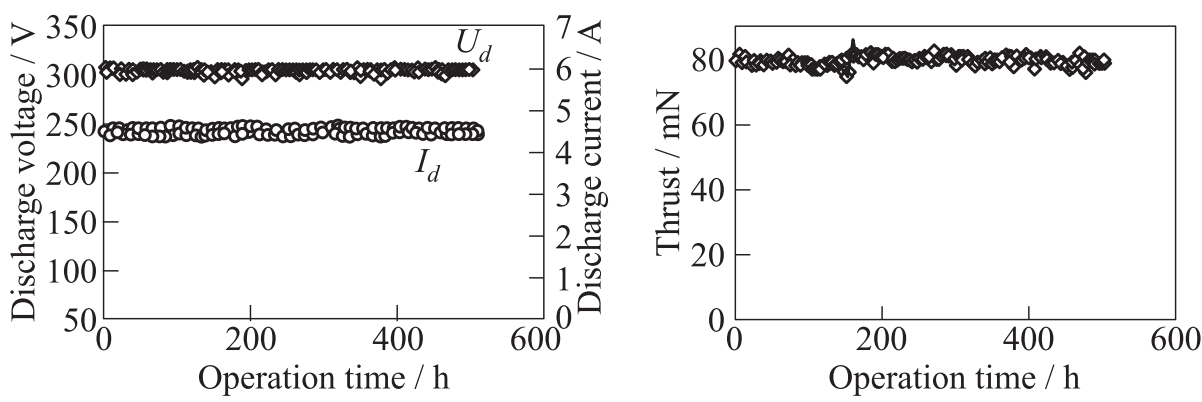

Figure 3 Evolution of parameters during life test 


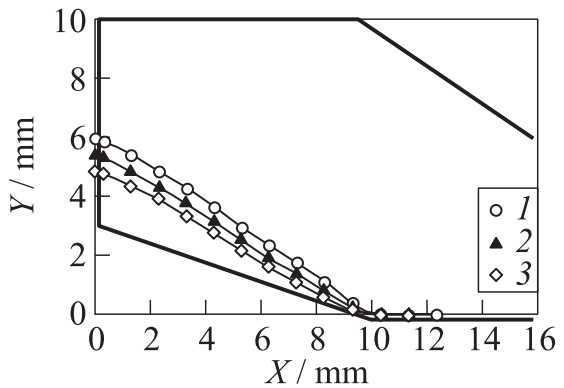

(a)

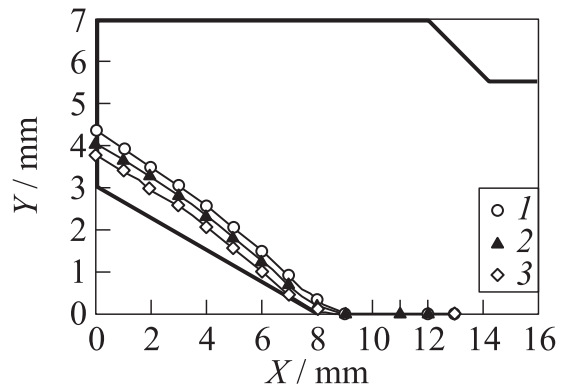

(b)

Figure 4 Insulator profile change (inner wall erosion $(a)$ and outer wall erosion $(b)$ ) during life test: $1-507 \mathrm{~h} ; 2-364$; and $3-250 \mathrm{~h}$

The rate of the inner wall erosion is much higher than that of the outer wall, so at a given thickness of the insulator walls near the channel exit, the lifetime is limited by the thickness of the inner wall.

Using the results of the 554-hour thruster operation and the procedure described in [4], the acceleration channel lifetime is estimated to be $\sim 6500-7000 \mathrm{~h}$.

\section{INTEGRATION OF THE PROPULSION UNIT WITH SPACECRAFT AND FLIGHT TESTS}

In the course of integration of the APU based on the KM-5 thruster into the "Express-A4" ("Express-A1P") SC, the following actions were performed:

- mechanical attachment of APU on the "Express-A4" SC, outside the pressurized container;

- xenon input from the standard station-keeping propulsion system into the GDU and further into the KM-5 thruster;

- creation of the electrical interface, including power supply of the thruster and GDU elements, transfer of control data from and telemetric data into the onboard telemetry system;

- taking away the generated heat.

A special matching and control unit was developed for power supply to, and control of, the KM-5 thruster. Since the power supply for the discharge circuit was provided by the standard power source, a special connector was included into the MCU to switch the discharge power from a spare DK4R thruster to the KM5 and back. As the power supply parameters required for KM-5 thruster, except 
the discharge power supply parameters, differ from that of the M100 thruster, the MCU was equipped with upgraded discharge current stabilization system, cathode heater current stabilizer, ignition system, and valve power source. The operation modes are defined according to analog telemetric data that is gathered by the MCU elements and transferred to the onboard computer system. The data includes discharge current and voltage, cathode heater current, and thermal throttle current. The MCU is developed based on the solutions used in the power processing unit (PPU), with the only difference that hermetically sealed contactors ("gerkons") are replaced with electromagnetic two-position relays. Since the MCU is integrated into the $\mathrm{SC}$ as an additional unit, it was installed onto the SC outside the pressurized container, close to the thruster and its only protection was provided by thermal radiation reflecting film. Autonomous thermal vacuum and mechanical tests proved the correctness of the aforementioned design schematics and technological solutions.

A block diagram of the electrical interface between the APU and "ExpressA4" SC systems is presented in Fig. 5 .

A pneumatic diagram of the APU units, pipelines, and their circuit connection with the standard station-keeping propulsion system (SKPS) is shown in

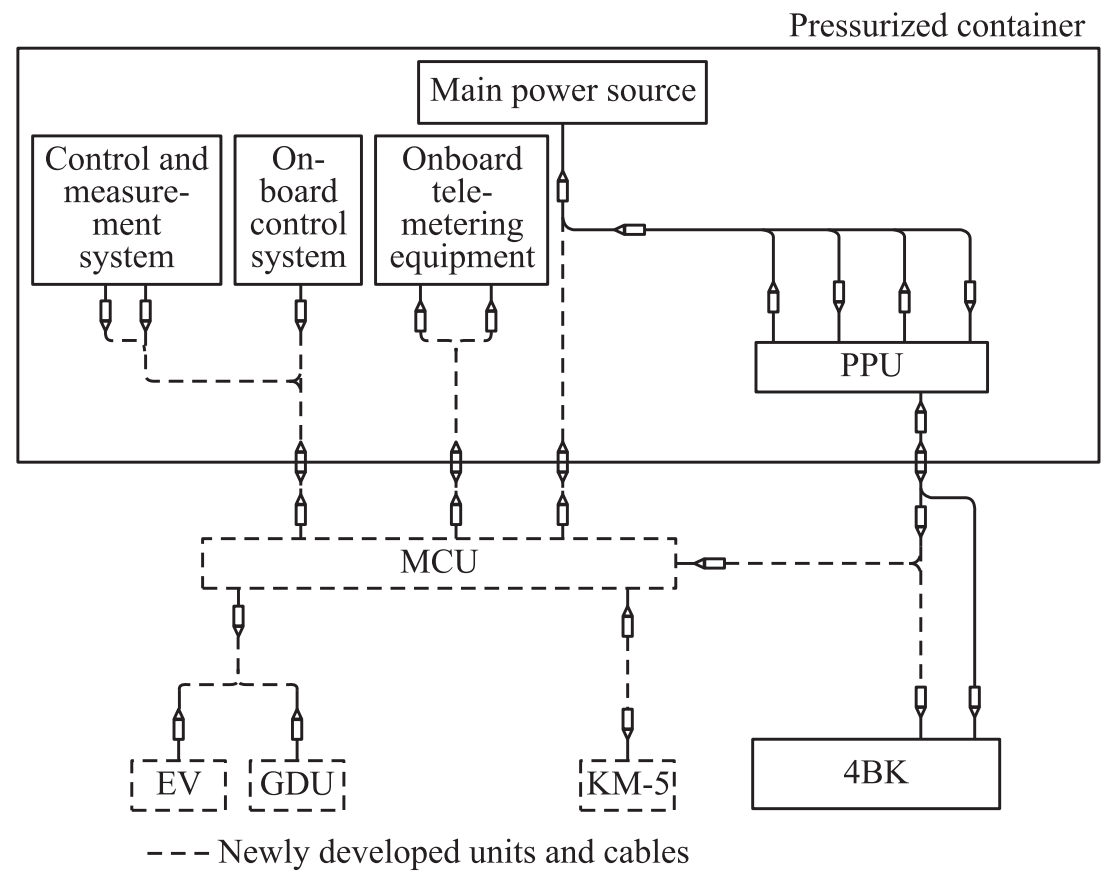

Figure 5 Block diagram of electrical interface and APU circuit connection 


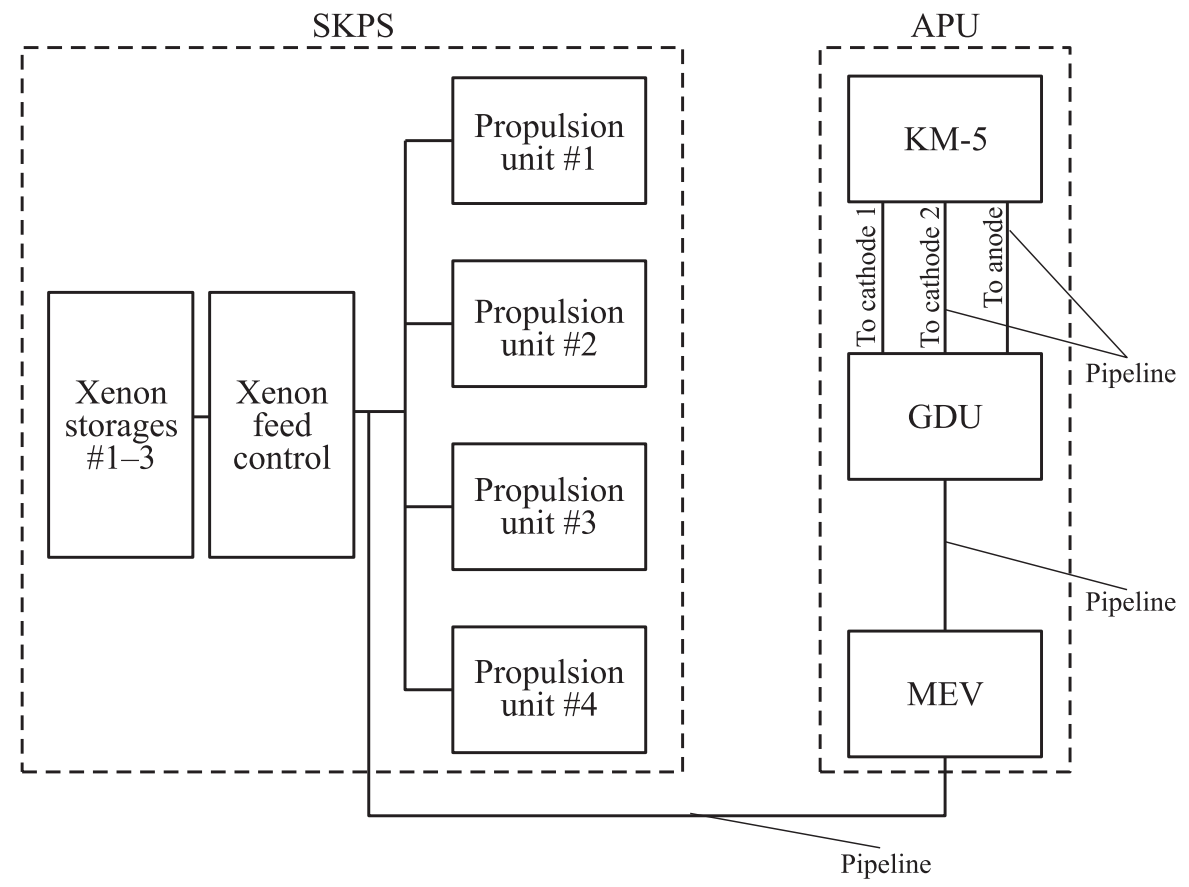

Figure 6 Diagram of APU and SKPS pneumatic circuit connection

A block diagram of the electrical interface between the APU and "ExpressA4" SC systems is presented in Fig. 5.

A pneumatic diagram of the APU units, pipelines, and their circuit connection with the standard station-keeping propulsion system (SKPS) is shown in Fig. 6. The APU utilizes xenon that is stored in the SKPS tanks. The same xenon is utilized by the standard M-100 thrusters.

A coupled firing test to prove the combined operability of the APU, namely, the KM-5 thruster, GDU, matching and control unit, and main electric valve (MEV), as well as to verify compatibility of the APU with the power processing unit (PPU), SKPS propellant subsystem and electric power system of the SC was performed in NPO PM. The test proved:

- electrical compatibility of MCU and PPU with the KM-5 thruster, GDU, and MEV;

- MCU and PPU combined operability;

- that the GDU and KM-5 thruster can operate while utilizing xenon from SKPS propellant subsystem; 


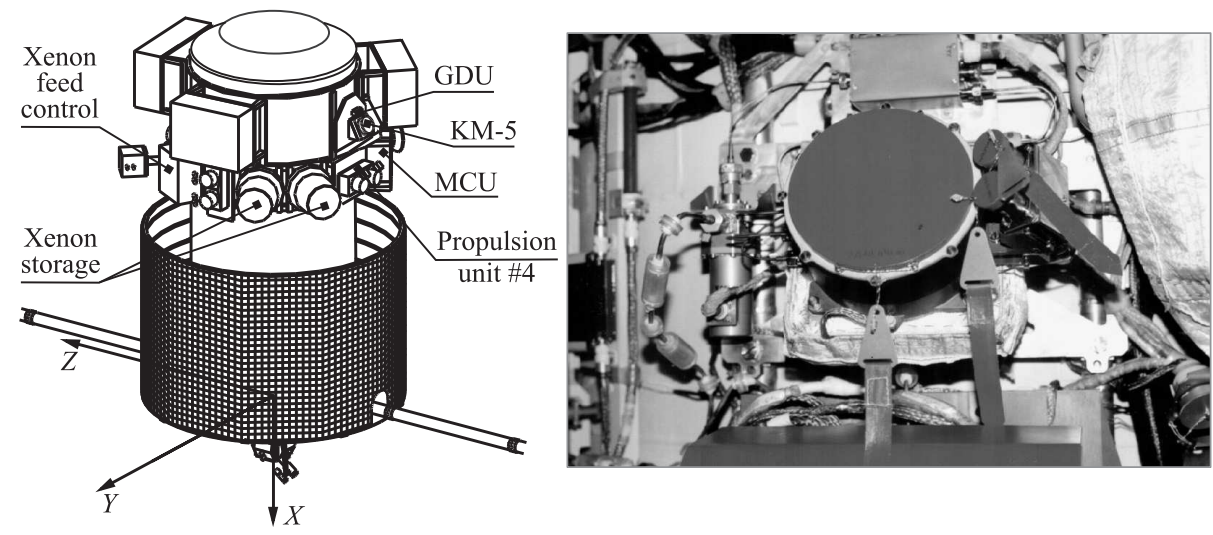

Figure 7 Additional propulsion unit equipment stationing onboard the "Express-A4" spacecraft

- reliable operation of PPU overload protection as the discharge current of the KM-5 thruster increased above the operating range;

- correctness of MCU control logic;

- KM-5 startup stability while it is performed by MCU and achievement of required discharge current stabilization accuracy;

- gathering of the required telemetric data; and

- APU operability while running with the SC power system. It was shown that $\mathrm{SC}$ power system $27-\mathrm{V}$ bus voltage pulsation magnitudes and levels of its transient processes are within the allowed limits.

The content of the APU mechanical interface test activities performed on the SC EM was as follows:

- harmonic vibration strength test;

- wideband random vibration strength test; and

- combined (electrical tests).

The APU electrical tests within the "Express-A4" SC included addressing checkup of control and telemetric circuits between the onboard integrated control unit (ICU) and the APU control unit. The APU stationing onboard the "Express-A4" SC is shown in Fig. 7.

The full-scale test phase of the KM-5 (T-120) thruster was started at the moment of "Express-A4" injection to GEO, on June 10, 2002. The APU preparation included evacuation of propellant feed pipelines, cathode outgassing, and 4 verification firings of the KM-5 thruster with different combinations of cathodes 
and xenon feed lines in the GDU. The total operation time during these firings was $25 \mathrm{~min}$ and $50 \mathrm{~s}$. All the startups and firings were performed according to the prescribed logic and without any failures. Reliable operation of the MCU during the flight test since June 2002 on board the SC proved the resistance to ionizing radiation of the elemental base used and that the design provides normal thermal conditions of electric and radio elements.

The KM-5 (T-120) thruster is now used for "Express-4A" orbit inclination keeping. No failures have been observed. The total operation time of the thruster to date (May 28, 2007) is $1074 \mathrm{~h}$ with 583 on/off cycles.

\section{CONCLUDING REMARKS}

1. KM-5 thruster, with 2-kilowatt nominal power, developed at $\mathrm{KeRC}$, is superior to all Hall thrusters already being used in space since it has a multimode operation capability and is capable of operating in the derated power mode $(1.35 \mathrm{~kW})$ and the enhanced power mode $(2.5 \mathrm{~kW})$.

2. Qualification activities have been performed for a propulsion unit composed of the KM-5 thruster and a GDU. The aforementioned propulsion unit was integrated into "Express-A4" SC.

3. The KM-5 thruster and GDU have been under continuous test since June, 2002. To date (May 28, 2007), the total operation time of the thruster is $1074 \mathrm{~h}$ at 583 on/off cycles. No failures have been observed.

4. KM-5 thruster application is a promising solution for such tasks as station keeping and orbit raising of medium and heavy class GEO spacecraft. It can also be efficient as a main engine of small interplanetary probes (particularly, for missions to the Moon and Mars).

\section{REFERENCES}

1. 2006 Commercial Space Transportation Forecasts. May 2006. Federal Aviation Administration FAA. Office of Commercial Space Transportation (AST) and Commercial Space Transportation Advisory Committee (COMSTAC).

2. Koroteev, A. S., O. A. Gorshkov, V.N. Akimov, A. A. Sinitsin, V.M. Murashko, B. A. Arkhipov, and V.N. Vinogradov. 2004. The status of electric propulsion development and applications in Russia. Acta Astronautica 54(1):25-37.

3. Vasin, A.I., O. A. Gorshkov, V.A. Muravlev, and A. A. Shagayda. 2005. Electric propulsion systems for the spacecraft: the state-of-the art in development and outlooks for progress. European Conference for Aerospace Sciences, EUCASS. Moscow.

4. Baranov, V.I., A.I. Vasin, A.A. Kalyev, and V.A. Petrosov. 1993. Prediction of electric thruster lifetime. 23th Electric Propulsion Conference (International) Proceedings. IEPC-93-099. 